\title{
Téoros
}

Revue de recherche en tourisme

\section{Le tourisme de masse au Mexique} Un virage?

\section{Daniel Hiernaux}

Volume 26, numéro 1, printemps 2007

Tourisme dans la Caraïbe : logiques régionales et perspectives de développement

URI : https://id.erudit.org/iderudit/1070990ar

DOI : https://doi.org/10.7202/1070990ar

Aller au sommaire du numéro

\section{Éditeur(s)}

Université du Québec à Montréal

\section{ISSN}

0712-8657 (imprimé)

1923-2705 (numérique)

Découvrir la revue

Citer cet article

Hiernaux, D. (2007). Le tourisme de masse au Mexique : un virage ? Téoros, 26(1), 15-20. https://doi.org/10.7202/1070990ar d'utilisation que vous pouvez consulter en ligne.

https://apropos.erudit.org/fr/usagers/politique-dutilisation/ 


\section{Le tourisme de masse au Mexique Un virage?}

\section{Daniel Hiernaux}

Cela fait plus de cinquante ans déjà que le Mexique a entrepris de développer son tourisme international autour d'un modèle massif, à l'image des développements connus en Europe du Sud et aux États-Unis. C'est autour de ce modèle massif que furent conçus les infrastructures, les programmes officiels de crédits à la construction hôtelière et les opérations touristiques depuis les années 1950 (Jiménez, 1992).

La position géographique du Mexique y est bien sûr pour beaucoup : la possibilité de profiter de l'ample marché des États-Unis, privé dans les années 1950 de son exutoire cubain, a joué un rôle décisif dans le développement du marché touristique mexicain. Toutefois, il ne faut pas oublier que le succès économique du pays - certains ont parlé de "miracle» pour l'équivalent mexicain des trente glorieuses mondiales -, a aussi contribué non seulement à l'engagement de capitaux mexicains privés et publics dans le tourisme, mais aussi au développement d'une demande nationale significative, qui a été le grand moteur des destinations traditionnelles comme Acapulco ou Veracruz.

Les crises mexicaines, mais aussi de l'économie mondiale et de son moteur principal, les États-Unis, n'ont fait qu'entamer le taux de croissance, sans changer radicalement la tendance à un tourisme toujours plus répandu et à l'apparition de nouvelles destinations tout le long des côtes mexicaines.

Toutefois, et cette hypothèse est le centre du présent article, on perçoit depuis quelques années une modification substantielle des tendances du tourisme international et national au Mexique, qui se traduit par des changements de marché, l'apparition de

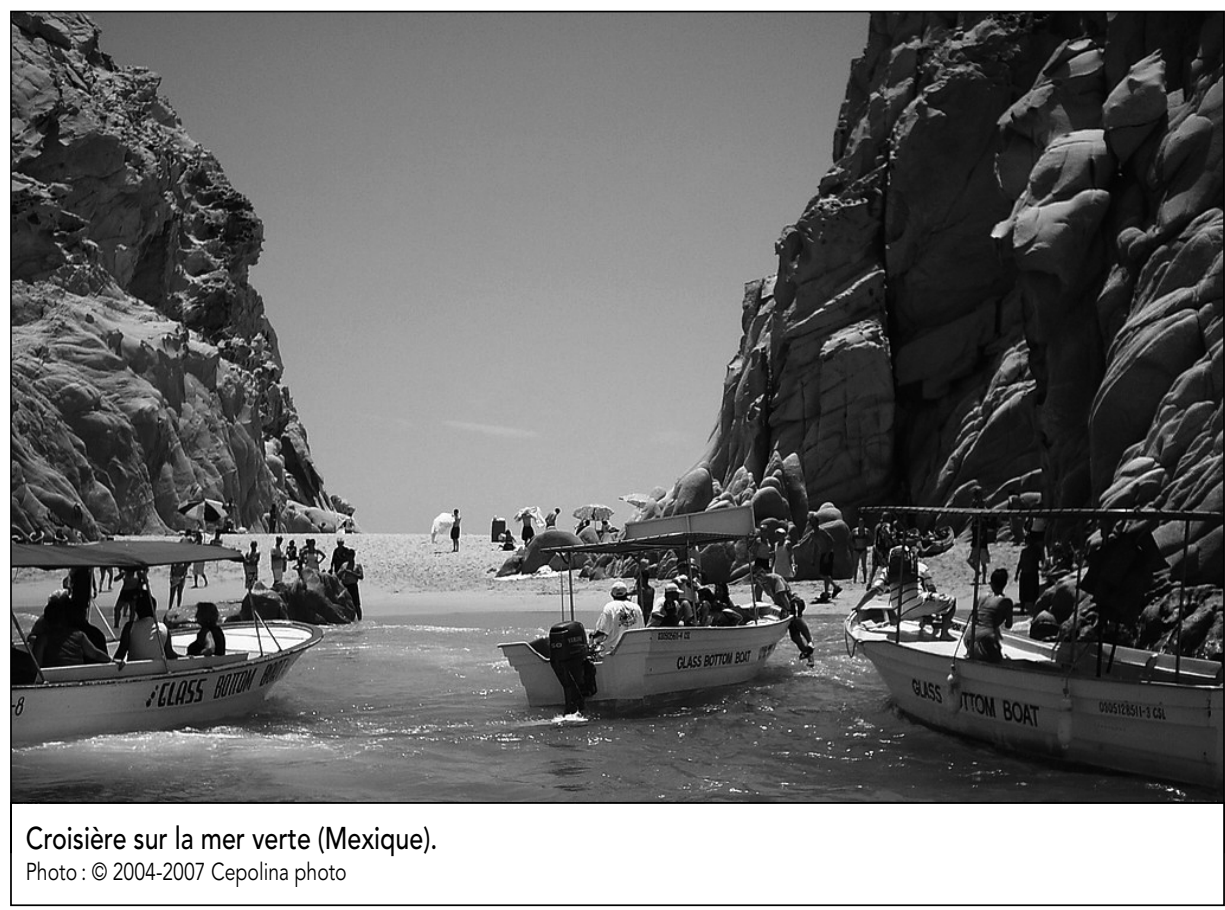

demandes différentes, la croissance de segments alternatifs d'offre; en synthèse des modifications assez puissantes des tendances antérieures sur le long terme, cela ne manque pas de faire grincer des dents des entrepreneurs traditionnels.

C'est à une brève synthèse de ces nouvelles orientations que nous nous attacherons dans les paragraphes qui suivent, après avoir passé en revue des tendances sur le long terme.

\section{Le Mexique : un leader touristique dans le tiers-monde}

Quelques indicateurs élémentaires et bien connus des spécialistes du tourisme situent les tendances générales du tourisme de masse au Mexique. Le Mexique se situe à la septième place des récepteurs de touristes internationaux (21,9 millions de touristes en 2005) et à la quatorzième place sur le plan des rentrées qui en proviennent (11,803 milliards USD en 2005). Soulignons aussi qu'en 2004 les activités liées au tourisme représentaient 7,7\% du produit intérieur brut $(\mathrm{PIB})$ du pays et que la génération d'emplois directs se situait autour de 1740000 unités, soit l'équivalent de 5,4\% de l'emploi national.

En soi, ces chiffres sont le reflet d'une situation très positive du tourisme dans l'activité économique mexicaine. Ils sont le résultat d'un développement touristique qui a commencé à avoir le vent en poupe durant la Seconde Guerre mondiale, le Mexique étant considéré comme une destination "sûre", qualificatif qui a repris aussi toute son importance après le 11 septembre 2001. 
En synthèse, on peut définir trois grandes étapes dans ce développement exemplaire: la première période qui est celle d'un développement spontané débutant dans les années 1940 du siècle dernier autour d'Acapulco, destination prisée du jet-set américain et international et de la nouvelle bourgeoisie mexicaine post-révolutionnaire (Hiernaux, 1994). La saturation de cette destination et la dégradation de ses infrastructures et de l'environnement, percevables dès les années 1960, poussa alors les autorités mexicaines à envisager de nouveaux projets soutenus par les ressources publiques, dont le premier, Cancun, débuta en 1976, suivi par Ixtapa, Los Cabos et Huatulco (Brenner, 2005 ; García de Fuentes, 1979 ; Hiernaux, 1999). Projets fortement publics dans une première phase, appuyés par une institution ad hoc, le Fondo Nacional de Fomento al Turismo (FONATUR) une sorte de corporation de développement touristique qui investit aussi directement dans les activités touristiques, ces nouvelles destinations prendront la tête des préférences du tourisme international, grâce entre autres à l'énorme battage publicitaire financé par le gouvernement, mais aussi à cause du déclin d'Acapulco et du manque d'appuis à d'autres destinations qui étaient pourtant prometteuses, Puerto Vallarta par exemple (Clancy, 2001).

La dernière phase est celle de la privatisation du modèle, avec une entrée en force des investissements étrangers, de nouveaux produits, une diversification des destinations, la politique de ciel ouvert avec l'autorisation des vols charters, entre autres aspects. Nous situons son démarrage en 1983, juste après la crise de 1982.

C'est à cette phase que nous nous attacherons dans la seconde partie de cet article, car c'est dans les vingt dernières années que vont poindre les nouvelles tendances que nous avons soulignées dans l'introduction.

\section{Nouvelles tendances, nouveau tourisme?}

Avant de présenter ces nouvelles tendances qui sembleraient révolutionner le tourisme de masse au Mexique, nous tenons à faire une précision sur le sens et l'orientation du modèle de masse dans les deux étapes antérieures.

\section{Tableau 1}

\begin{tabular}{l|ccc|cc}
\multicolumn{7}{c}{ Arrivées de touristes au Mexique, 1993-2005 } \\
Année & $\begin{array}{c}\text { Touristes } \\
\text { intérieurs }\end{array}$ & $\begin{array}{c}\text { Touristes } \\
\text { frontaliers }\end{array}$ & $\begin{array}{c}\text { Touristes } \\
\text { totaux }\end{array}$ & $\begin{array}{c}\text { Intérieur/ } \\
\text { total }\end{array}$ & $\begin{array}{c}\text { Taux de } \\
\text { croissance } \\
\text { annuelle }\end{array}$ \\
\cline { 2 - 5 } & \multicolumn{3}{|c}{ (milliers detouristes) } & $\%$ & $\%$ \\
\hline 1993 & 6625 & 9815 & 16440 & 40,30 & \\
1994 & 7135 & 10047 & 17182 & 41,53 & 4,51 \\
1995 & 7784 & 12457 & 20241 & 38,46 & 17,80 \\
1996 & 8982 & 12413 & 21395 & 41,98 & 5,70 \\
1997 & 9794 & 9557 & 19351 & 50,61 & $-9,55$ \\
1998 & 9775 & 9617 & 19392 & 50,41 & 0,21 \\
1999 & 10214 & 8829 & 19043 & 53,64 & $-1,80$ \\
2000 & 10591 & 10050 & 20641 & 51,31 & 8,39 \\
2001 & 10151 & 9659 & 19810 & 51,24 & $-4,03$ \\
2002 & 9883 & 9784 & 19667 & 50,25 & $-0,72$ \\
2003 & 10353 & 8312 & 18665 & 55,47 & $-5,09$ \\
2004 & 11553 & 9065 & 20618 & 56,03 & 10,46 \\
2005 & 12564 & 9381 & 21915 & 57,33 & 6,29 \\
\hline
\end{tabular}

Source : Secretaria de Turismo, 2006 ; et Datatur, calculs personnels.
S'il est vrai que le tourisme résidentiel est apparu dès les premières années du développement touristique mexicain, c'est un tourisme essentiellement hôtelier qui a été appuyé par les secteurs privé et public et qui forme le noyau dur du modèle mexicain : ni données concrètes sur les résidences touristiques, ni appuis envers ce type de produits $^{1}$, le modèle mexicain s'est ainsi échafaudé autour du développement de grands complexes hôteliers, fonctionnant selon des logiques d'organisation du travail et de pro-

\section{Carte 1}

\section{Villes principales et destinations touristiques au Mexique}

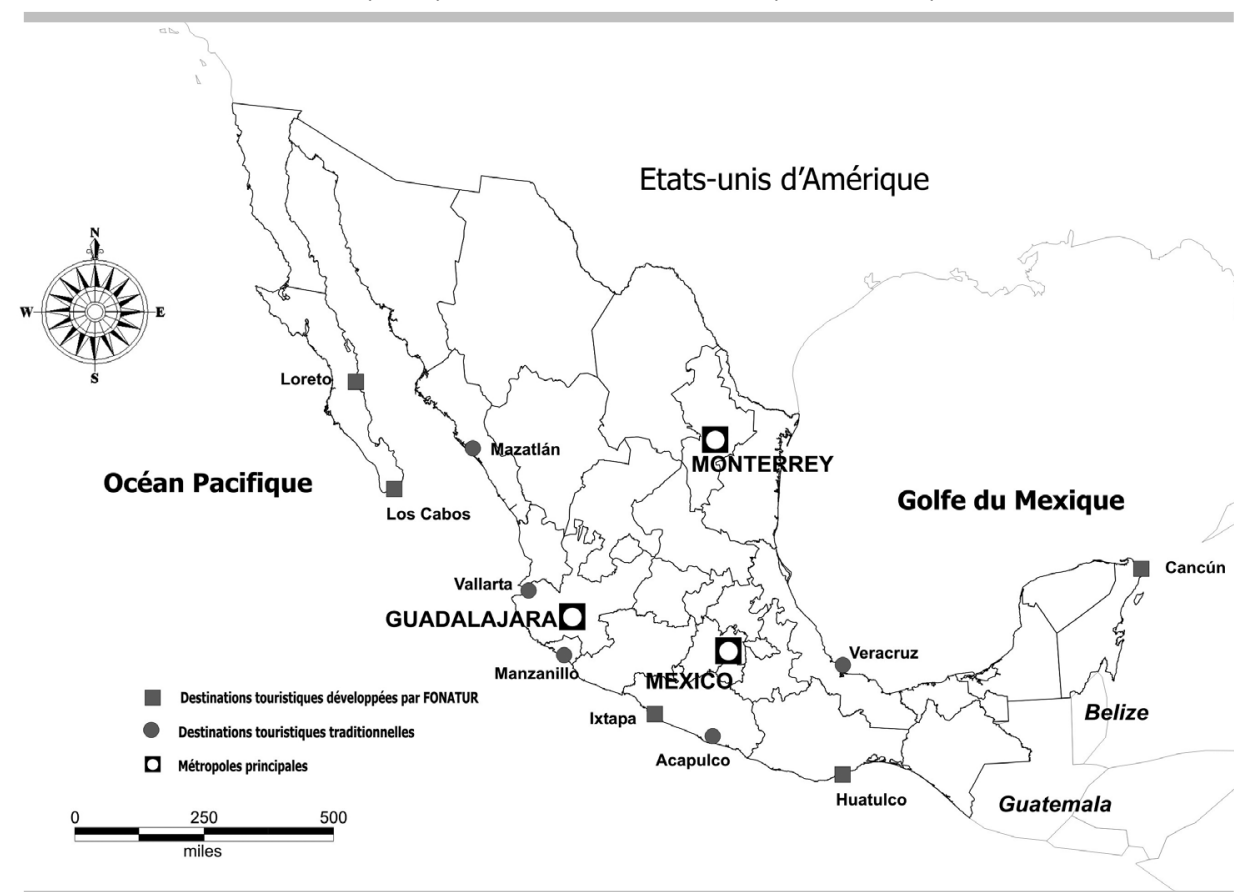

Source : Élaboration personnelle. 
duction qui s'inspirent des principes du taylorisme appliqués à l'industrie. II en résulte un tourisme massif, encapsulé dans des bulles touristiques, appuyé par l'État qui génère des produits touristiques semblables dans tout le pays et toutes les installations, soit un modèle touristique que plusieurs auteurs ont qualifié de "fordiste" (Hiernaux, 2003 ; Torres et Momsen, 2005).

La capacité même de croissance de ce modèle n'était pas seulement liée à la demande croissante du tourisme international, mais aussi à l'offre, celle-ci étant à son tour conditionnée par les interventions publiques qui déterminèrent ainsi les variables principales du modèle : les localisations, la qualité des installations (par le biais de crédits préférentiellement orientés vers les installations de plus de trois étoiles) (Brenner et Aguilar, 2002), le contrôle des travailleurs (par l'entremise de syndicats dociles et contrôlés par l'État) et le type d'activités de récréation permises et soutenues (pas de casino ni de jeux de hasard, par exemple).

II n'est donc pas difficile de comprendre que le retrait progressif de l'État mexicain après la crise économique majeure de 1982 a ouvert la porte à toutes sortes de modifications de ce modèle, passant progressivement d'un "fordisme d'État périphérique " à un "post-fordisme» croissant. Ce qui n'empêche pas que le gouvernement mexicain ait encore des capacités certaines pour marquer l'orientation du modèle; mais celui-ci est davantage déterminé par le marché en accord avec les entrepreneurs mexicains et les groupes internationaux que par le passé.

En premier lieu, il faut souligner que le marché s'est diversifié quant aux destinations touristiques: dès les années 1980, de nombreux projets ont été proposés par des groupes d'entrepreneurs nationaux, internationaux et mixtes ${ }^{2}$. Beaucoup de ceux-ci étaient destinés à relancer des destinations en souffrance, dont Acapulco, d'autres à appuyer des sites encore insuffisamment développés, dont Puerto Vallarta, Mazatlán ou divers endroits de la côte de la Basse-Californie. Soulignons d'ailleurs que les destinations soutenues par FONATUR ont eu un succès restreint, sauf dans les cas de Cancun et sa dérivation postérieure, la côte du Quintana Roo (Torres et Momsen, 2005 ; Hiernaux, 1999), la «Riviera Maya » et, d'une certaine façon, Los Cabos.

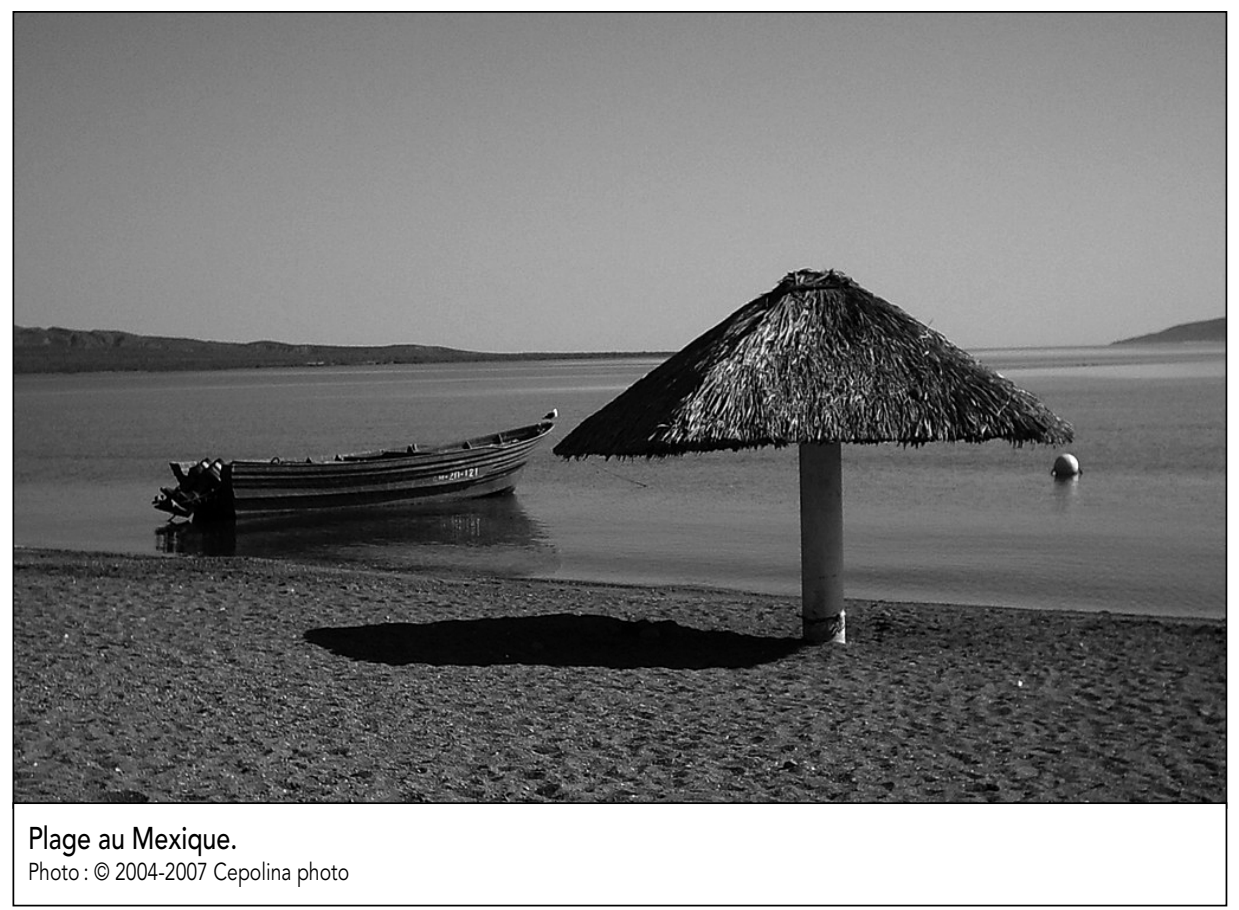

Ces nouveaux projets correspondent aussi à de nouvelles orientations de marché : en premier lieu, dans les années 1980 et 1990, les «temps partagés » ont connu un immense succès, au point que de nombreux hôtels ont été réaménagés en ce sens. On voit déjà, dans ce cas, poindre des intérêts immobiliers qui ont pris une ampleur sans précédent dans les dernières années.

On a assisté aussi à une diversification des chaînes hôtelières qui opèrent dans le pays : cela est dû à l'amélioration du climat envers les entreprises, moins soumises aux impératifs du gouvernement que par le passé, mais aussi aux investissements privés en quête d'opérateurs connus dans les marchés internationaux. Une partie des capitaux ont afflué de l'étranger (entre autres par des échanges de dettes). Les entreprises mexicaines ont investi aussi dans ces nouveaux projets, tout particulièrement celles qui ont fait de grands bénéfices pendant le boom pétrolier et par la suite l'ouverture des marchés et qui ont décidé de s'inscrire dans le mouvement de forte croissance du tourisme après la crise de $1982^{3}$. Finalement, il ne faut pas négliger la croissance de la participation de capitaux à la recherche de «blanchiment $»^{4}$.

Une autre tendance forte est l'amélioration de la qualité des services : ceux-ci sont restés très longtemps dans la dynamique fordiste (produits massifs à bas prix mais de basse qualité) pour s'insérer dans la nouvelle recherche de qualité, liée à une meilleure prise en compte du client : non seulement les grands hôtels ont-ils amélioré leurs services et la qualité des installations, mais on a assisté aussi au développement de petites unités hôtelières du genre "hôtel boutique ", appart-hôtels ${ }^{5}$, etc. II y a donc une diversification accrue du marché, ce qui est favorable à la demande.

Non seulement a-t-on pu observer des changements liés à l'opération même de l'offre et aux localisations, mais on a aussi assisté à une modification des imaginaires touristiques dans les dernières années (Hiernaux, 2002). Le modèle avion-hôtelplage reste pourtant dominant dans la demande, mais il est de plus en plus soumis à la concurrence de nouvelles aspirations des touristes liées, entre autres, à la culture ou au retour à la nature.

Parmi celles-ci le tourisme urbain est en pleine croissance. Certes il peut paraître difficile de discerner le tourisme d'affaires, dont la forte croissance est en relation avec la mondialisation et en particulier la nouvelle dynamique des trois économies de l'Amérique du Nord dans le cadre de l'accord de libre-échange nord-américain - ALENA - du " véritable " tourisme vers les villes, mais il est certain que celui-ci prend de la vitesse: à l'heure actuelle, les destinations urbaines 
Tableau 2

Tendances du tourisme par destination, 2001-2003 (nombre total de touristes par année)

\begin{tabular}{|c|c|c|c|c|c|}
\hline Destination touristique & type de destination & 2001 & 2003 & $\%$ total 2003 & TMCA 01-03 \\
\hline Total toutes destinations & & 37629502 & 38124044 & 100,00 & 0,65 \\
\hline Campeche & & 234803 & 242105 & 0,64 & 1,54 \\
\hline Guanajuato & & 469720 & 398684 & 1,05 & $-7,87$ \\
\hline Morelia & & 746608 & 819389 & 2,15 & 4,76 \\
\hline Oaxaca & & 686275 & 826218 & 2,17 & 9,72 \\
\hline Pachuca & & 233009 & 230023 & 0,60 & $-0,64$ \\
\hline Querétaro & & 614530 & 647836 & 1,70 & 2,67 \\
\hline San Cristobal de las Casas & & 273069 & 373339 & 0,98 & 16,93 \\
\hline Tlaxcala & & 93214 & 127135 & 0,33 & 16,79 \\
\hline Zacatecas & & 465167 & 409415 & 1,07 & $-6,18$ \\
\hline Sous-total & culture & 3816395 & 4074144 & 10,69 & 3,32 \\
\hline Aguascalientes & & 518099 & 512388 & 1,34 & $-0,55$ \\
\hline Chihuahua & & 494931 & 513596 & 1,35 & 1,87 \\
\hline Colima & & 180751 & 143484 & 0,38 & $-10,90$ \\
\hline Durango & & 254667 & 265815 & 0,70 & 2,17 \\
\hline Hermosillo & & 514325 & 592379 & 1,55 & 7,32 \\
\hline La Paz & & 305570 & 280963 & 0,74 & $-4,11$ \\
\hline León & & 1072075 & 800316 & 2,10 & $-13,60$ \\
\hline San Juan del Río & & 136601 & 119773 & 0,31 & $-6,36$ \\
\hline San Luis Potosi & & 543797 & 563407 & 1,48 & 1,79 \\
\hline Tepic & & 136928 & 262801 & 0,69 & 38,54 \\
\hline Toluca & & 125382 & 185047 & 0,49 & 21,49 \\
\hline Tuxtla Gutiérrez & & 476133 & 598991 & 1,57 & 12,16 \\
\hline Villahermosa & & 540767 & 615376 & 1,61 & 6,68 \\
\hline Sous-total & économie & 5300026 & 5454336 & 14,31 & 1,45 \\
\hline Ciudad Juárez & & 1150241 & 988125 & 2,59 & $-7,31$ \\
\hline Tijuana & & 926897 & 1042881 & 2,74 & 6,07 \\
\hline Sous-total & frontière & 2077138 & 2031006 & 5,33 & $-1,12$ \\
\hline Destination touristique & & 2001 & 2003 & $\%$ total & TMCA 87-01 \\
\hline Distrito Federal & & 9486499 & 9524728 & 24,98 & 0,20 \\
\hline Guadalajara & & 1977274 & 1930119 & 5,06 & $-1,20$ \\
\hline Monterrey & & 1479341 & 1456797 & 3,82 & $-0,76$ \\
\hline Puebla & & 1204979 & 1478020 & 3,88 & 10,75 \\
\hline Sous-total & métropole & 14148093 & 14389664 & 37,74 & 0,85 \\
\hline Acapulco & & 2196747 & 1724624 & 4,52 & $-11,40$ \\
\hline Costa Alegre & & 313548 & 241042 & 0,63 & $-12,32$ \\
\hline Manzanillo & & 624783 & 712142 & 1,87 & 6,76 \\
\hline Mazatlán & & 833716 & 726685 & 1,91 & $-6,64$ \\
\hline Playas de Rosarito & & 682510 & 526649 & 1,38 & $-12,16$ \\
\hline Puerto Vallarta & & 1203304 & 1132556 & 2,97 & $-2,98$ \\
\hline Veracruz & & 1489370 & 1693103 & 4,44 & 6,62 \\
\hline Sous-total & plage tradition & 7343978 & 6756801 & 17,72 & $-4,08$ \\
\hline Bahias de Huatulco & & 206064 & 255007 & 0,67 & 11,24 \\
\hline Cancún & & 2986489 & 3057327 & 8,02 & 1,18 \\
\hline Ixtapa Zihuatanejo & & 412144 & 710985 & 1,86 & 31,34 \\
\hline Loreto & & s.d & s.d. & & \\
\hline Los Cabos & & s.d. & s.d. & & \\
\hline Sous-total & plage planifiée & 3604697 & 4023319 & 10,55 & 5,65 \\
\hline Cuautla & & 207766 & 174545 & 0,46 & $-8,34$ \\
\hline Cuernavaca & & 495200 & 573205 & 1,50 & 7,59 \\
\hline Ixtapan de la Sal & & 84391 & 127567 & 0,33 & 22,95 \\
\hline San Miguel de Allende & & 211880 & 200032 & 0,52 & $-2,84$ \\
\hline Taxco & & 167837 & 163018 & 0,43 & $-1,45$ \\
\hline Tequisquiapán & & 80182 & 78429 & 0,21 & $-1,10$ \\
\hline Valle de Bravo & & 91919 & 77978 & 0,20 & $-7,89$ \\
\hline Sous-total & résidentiel & 1339175 & 1394774 & 3,66 & 2,05 \\
\hline \multicolumn{6}{|c|}{$\begin{array}{l}\text { Sources }: \text { Datatur } ; \text { sans données } ; \text { TMCA = taux moyen de croissance annuelle. } \\
\text { Les destinations touristiques sont classifiées selon leurs activités prédominantes. } \\
\text { Culture }=\text { ville patrimoine, destination culturelle } ; \text { économie }=\text { ville moyenne } ; \text { frontière = ville frontalière; } \\
\text { métropole }=\text { les } 4 \text { grandes métropoles mexicaines } ; \text { plage tradition = destination traditionnelle de plage; } \\
\text { plage planifiée = destination planifiée par le FONATUR; résidentiel = destination principalement de tourisme résidentiel. }\end{array}$} \\
\hline
\end{tabular}

captent plus de touristes que les destinations de plage. Derrière cela on peut distinguer une tendance mondiale vers des séjours plus courts, soit des véritables coupures envisagées comme des moments de répit face aux pressions de la vie postmoderne. Mais il s'agit aussi d'un changement dans les imaginaires touristiques, de plus en plus lié à la culture et en particulier à deux aspects centraux: la recherche de destinations ou de circuits culturels autour du patrimoine bâti (particulièrement important au Mexique surtout dans le centre et le sud-est du pays), mais aussi le fait d'apprécier davantage les styles de vie urbains, qui attirent les touristes vers les centres historiques, les zones piétonnes, les petits commerces urbains et la terrasse des cafés, plus que la plage et les cocotiers. Toutefois, il faut bien reconnaitre que cette tendance est surtout l'apanage des groupes urbains plus «illustrés », davantage "postmodernes », peu attirés par les destinations de plage les plus massifiées qui continuent à être fréquentées par les secteurs aux ressources plus modestes, aussi bien mexicains qu'étrangers.

La croissance des préoccupations environnementales dans tous les secteurs ne pouvait pas épargner le tourisme: le modèle touristique fordiste, grand pollueur, a été réticent à suivre ce mouvement, mais la législation durcie, tout comme les demandes même des clients, ont poussé progressivement les entreprises touristiques à introduire une certaine forme d'«intérêt écologique restreint » dans leur mode d'opération. Le tourisme de masse est aussi soumis à la croissance de segments alternatifs, dont le tourisme dit "écologique » ou "durable » qui s'empare d'une proportion encore restreinte, mais croissante du marché touristique. Toutefois, la multiplication des projets "alternatifs" ne démontre pas un grand succès et on estime à moins de $10 \%$ la demande qui se concentre sur ces projets.

La tendance la plus importante a eu lieu dans les dernières années et est susceptible de modifier radicalement le paysage touristique mexicain : il s'agit de la croissance massive du tourisme résidentiel. II y a, d'une part, la croissance du tourisme résidentiel de long séjour (plus de trois mois, souvent de "snowbirds ", soit des retraités qui passent l'hiver au Mexique) ; d'autre part, on assiste à une certaine démocratisation de l'accès à la seconde résidence en faveur de la classe moyenne mexicaine. 
Les motifs des deux versants de cette croissance sont multiples. Dans le premier cas, celui des secondes résidences pour longs séjours, la croissance résulte en premier lieu d'une demande accrue : les analystes ont pointé du doigt l'existence de la demande des retraités, en pleine croissance au vu du vieillissement de la population. On compte plus de 40 millions de personnes retraitées aux États-unis, chiffre qui atteindra 70 millions dans 20 ans (Youden, 2006). Ces personnes dépensent moins pour entretenir leur seconde résidence toute l'année et payer les impôts mexicains (les taxes locales, dont la taxe d'habitation) que ce que leur coûte cette dernière en Californie, sans parler de la différence du coût de la vie et de la qualité du climat. Mais ce ne sont pas seulement les «baby-boomers » qui sont pointés du doigt: leurs enfants, qui ont fréquenté les destinations touristiques mexicaines dès leur enfance, cherchent aussi à acquérir une seconde résidence. Le facteur sécurité est aussi décisif, comme nous le mentionnions plus haut, Mexico est encore considéré comme une destination sûre après le 11 septembre 2001, même si les problèmes d'insécurité ont considérablement augmenté dans plusieurs destinations (dont Acapulco, du fait des conflits territoriaux entre plusieurs cartels de la drogue).

On estime actuellement que $7.6 \%$ du tourisme international vers le Mexique se loge dans des résidences louées ou achetées et pas à l'hôtel (Hiernaux, 2004). Les Mexicains ne sont pas non plus en reste : ils sont eux aussi friands de seconde résidence, soit dans des destinations touristiques traditionnelles ou nouvelles, soit dans des villes secondaires et des villages autour des métropoles. Ce serait $8.5 \%$ des Mexicains qui possèdent ou louent une seconde résidence (ibid, ; SECTUR-INEGI, 2003).

L'offre de secondes résidences était relativement limitée jusque dans les années récentes. II s'agissait surtout d'initiatives individuelles ou de lotissements développés par des promoteurs, la construction de la maison restant aux soins de l'acheteur. Aujourd'hui, une offre clé sur porte s'impose sur le marché, autant pour les résidences individuelles (dans des complexes de condominiums horizontaux) que pour les appartements. Cette augmentation de l'offre est aussi due à une série de politiques sur le logement qu'il serait trop long de développer

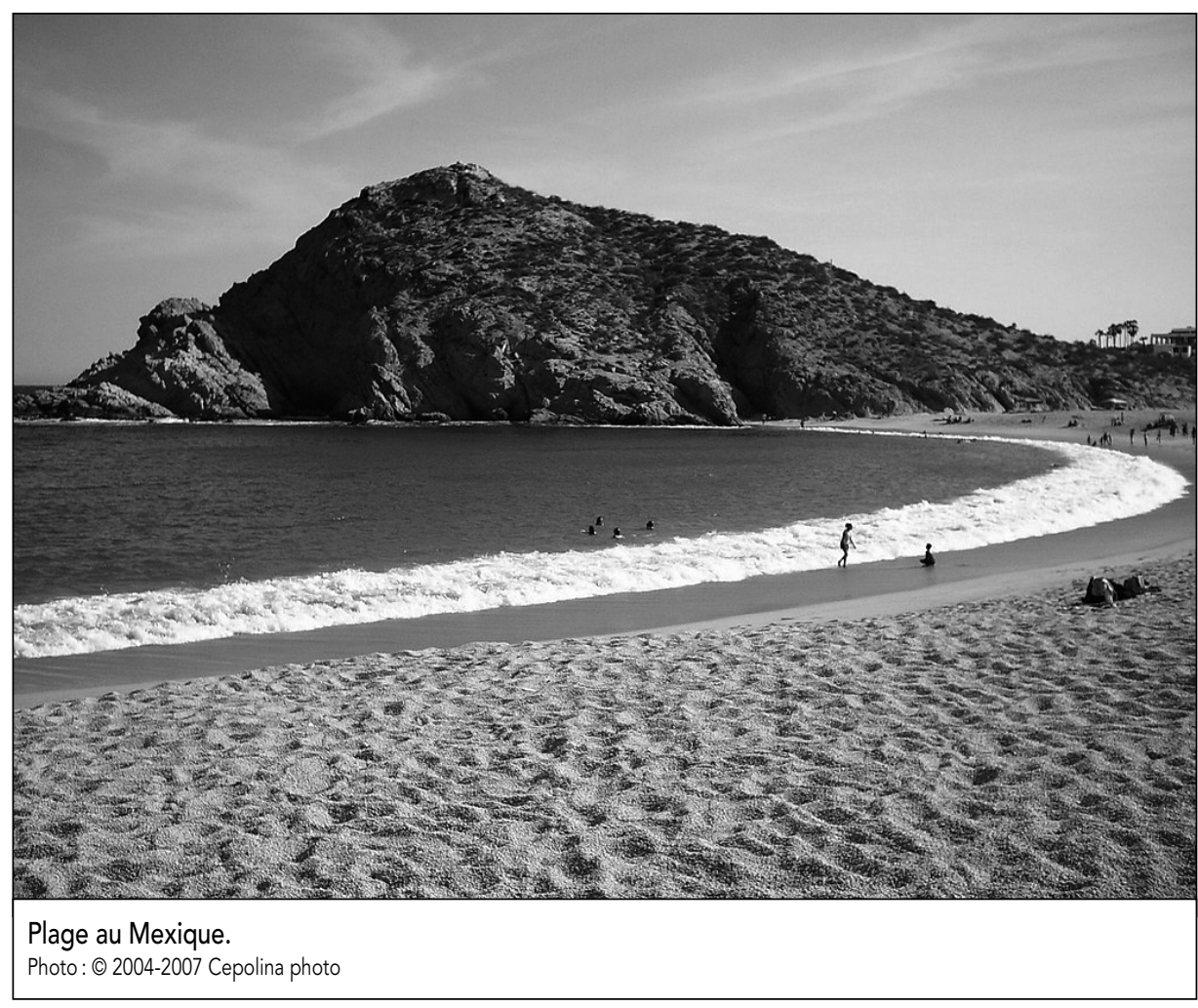

ici, mais qui ont consisté essentiellement en l'ouverture de crédits favorables au développement immobilier, tous produits confondus (Secretaría de Desarrollo Social, 2005).

Le développement de complexes importants de secondes résidences, joue aussi un rôle décisif pour assurer la sécurité des résidants (les complexes sont généralement fermés, sans pour autant être tout à fait des gated communities à la californienne) ; il peut y avoir une administration centrale qui joue le rôle d'agent immobilier informel (sans déclarer les transactions aux autorités fiscales) pour louer les résidences le reste de l'année quant le propriétaire ne l'occupe pas.

Finalement, notons aussi que de nombreux autres pays de l'Amérique latine, longtemps en retard quant au développement de leur tourisme en comparaison avec le Mexique, ont compris que le tourisme international pouvait être un stimulant de leur économie, difficilement industrialisable à grande échelle (Cazes, 1992) ; ils profitent aussi des expériences internationales, soit pour imiter en quelque sorte le cas mexicain (Cuba, République dominicaine...), soit pour essayer d'éviter les erreurs d'un tourisme de masse fordiste aujourd'hui plutôt récrié internationalement.
C'est ainsi que des pays comme le Costa Rica misent fortement sur un tourisme durable, alors que l'Argentine revalorise par le tourisme le Sud patagonique que les politiques d'industrialisation des années 1980 n'avaient pu développer suffisamment. Le Chili semble choisir une direction semblable et tous ces nouveaux développements ne manquent pas de se placer en concurrence directe avec le modèle mexicain, qui n'a encore entrepris qu'un virage partiel et n'a pas encore osé rectifier drastiquement ses politiques officielles d'appui au tourisme, même si certaines initiatives vont dans le bon sens.

Le Mexique se trouve donc face à un dilemme : continuer sur la trace du développement hôtelier massif qui a fait ses beaux jours par le passé (mais au prix d'une diminution de la qualité de la demande comme c'est déjà le cas à Cancun soumis à une demande certes massive, mais peu dépensière, ou même destructrice, les springbreakers nord-américains par exemple ${ }^{6}$ ), ou s'engager plus radicalement sur les nouvelles voies qui s'ouvrent dans le monde entier, comme le tourisme d'aventure, l'écotourisme (sur la côte d'Oaxaca, dans le Chiapas, les destinations se sont multipliées), le tourisme culturel, etc. La massifi- 
cation a certes porté fruits, mais elle devrait s'effacer pour construire un tourisme plus diversifié et surtout plus équitable et durable (Hiernaux, 2006).

Daniel Hiernaux est géographe, professeur-chercheur titulaire du Département de sociologie et du programme de géographie humaine de l'Université autonome métropolitaine, campus Iztapalapa de Mexico.

\section{Notes}

1 Alors que des pays comme le Canada ou la France identifient bien les logements qui ne sont utilisés que temporairement en tant que seconde résidence, le Mexique n'a pas encore compris l'importance de pouvoir quantifier cet indicateur. II n'y a donc que des estimations très incomplètes du processus de croissance des résidences secondaires. Par ailleurs, tous les appuis en matière de crédits au développement et de promotion touristique se sont essentiellement orientés vers les stations balnéaires et le tourisme hôtelier.

2 Ces projets ont été appelés «méga projets" car l'échelle des infrastructures et des produits était très supérieure aux actions ponctuelles entreprises par le capital privé par le passé; un bon exemple de méga projet est Puerto Aventuras, sur le littoral caribéen du Mexique au sud de Cancun. D'autres ont été proposés le long des côtes mexicaines, essentiellement sur le Pacifique, la mer Caraïbe et rarement le golfe du Mexique.

3 Les entreprises de Bâtiment Travaux Publics ont connu des heures de gloire pendant le boom pétrolier et ont été les premières à s'associer aux grandes chaînes hôtelières pour développer de nouveaux projets dont le succès était garanti en raison de la forte dévaluation de 1982 qui augmenta radicalement la compétitivité du Mexique en matière de tourisme international.

4 Nous faisons référence ici aux capitaux de la drogue, dont le marché a considérablement augmenté après 1982; les grands cartels ont trouvé dans les activités touristiques un débouché important pour blanchir les énormes capitaux qu'ils accumulent; même si le gouvernement prétend minimiser ce fait, les informations de presse quant à la saisie de biens de trafiquants arrêtés démontrent bien leur participation dans les activités touristiques, particulièrement dans l'hôtellerie.
5 Les « appart-hôtels » sont des appartements de particuliers ou de petites entreprises qui sont organisés pour recevoir des touristes: ils y disposent non seulement de toutes les facilités propres à une résidence (cuisine, télévision, Internet, etc.), mais aussi de services hôteliers (gardiennage, nettoyage des chambres, etc.). Cette modalité de logement touristique est très prisée par les voyageurs d'affaires, mais aussi par des personnes qui préfèrent ce type de logement plus commode et moins cher que les hôtels, auxquels ils font une très forte concurrence dans les grandes villes. Les «appart-hôtels » se développent peu à peu au Mexique, mais sont déjà bien plus développés dans les grandes métropoles latino-américaines, dont Santiago au Chili, Buenos Aires ou Sao Paulo.

6 Les spring-breakers sont des groupes de jeunes qui achètent hors saison (soit à la fin de l'hiver) des offres à très bas prix dans certaines stations balnéaires, Cancun en particulier. Ces véritables « hordes dorées ", pour reprendre l'expression de Turner et Ash (1991), violent toutes les règles traditionnelles du tourisme de masse, plutôt bon enfant, par des orgies d'alcool, de drogue et de sexe en public, sans compter des destructions de mobilier urbain et même des dégâts dans les installations touristiques. Ils sont tolérés par les autorités locales et, d'une certaine façon, stimulés par les politiques touristiques et les entrepreneurs touristiques qui préfèrent des taux élevés d'occupation hôtelière aux dépens de la qualité (et même de la respectabilité) du modèle touristique.

\section{Bibliographie}

Brenner, Ludger (2005), « State-planned Tourism Destinations: The Case of Huatulco, Mexico", Tourism Geographies, vol. 7, $n^{\circ} 2$, p. 138-164.

Brenner, Ludger, et Guillermo A. Aguilar (2002), "Luxury Tourism and Regional Economic Development in México ", The Professional Geographer, vol. 54, n 4, p. 500-520.

Cazes, Georges (1992), Tourisme et TiersMonde, un bilan controversé : Les nouvelles colonies de vacances? tome II, Paris, L'Harmattan, collection "Tourismes et Sociétés ».

Clancy, Michael (2001), "Mexican Tourism: Export Growth and Structural Change since 1970 ", Latin American Research Review, vol. 36, no 1, p. 128-150.

García de Fuentes, Ana (1979), Cancún: Turismo y subdesarrollo regional, México, Universidad Nacional Autónoma de México, Serie Cuadernos.
Hiernaux, Daniel (1994), «En busca del Edén: Turismo y territorio en las sociedades modernas ", Ciudades, Puebla, Red Nacional de Investigación Urbana, p. 24-31.

Hiernaux, Daniel (1999), "Cancun Bliss", dans Dennis Judd et Susan Fainstein (dir.), Tourist Cities, Yale University Press, New Haven, p. 125-139.

Hiernaux, Daniel (2002), "Turismo e imaginarios ", dans Daniel Hiernaux, Allen Cordero et Luisa Van Duynen Montijn (dir.), Imaginarios sociales y turismo sostenible, San José de Costa Rica, Facultad Latinoamericana de Ciencias Sociales / FLACSO, p. 7-36.

Hiernaux, Daniel (2003), « Mexico: Tensions in the Fordist Model of Tourism Development ", dans Lily M. Hoffman, Susan S. Fainstein et Dennis R. Judd (dir.), Cities and Visitors, Regulating People, Markets and City Space, Oxford (Angleterre), Blackwell Publishing, p. 187-199.

Hiernaux, Daniel (2004). El turismo de segundas residencias en México, México, Universidad Autónoma Metropolitana Iztapalapa, Secretaría de Turismo.

Hiernaux, Daniel (2006), "Tourisme au Mexique: Modèle de masse, de l'étatisme au marché ", Alternatives Sud, vol. 13, p. 99-214.

Jiménez, Alfonso (1992), Turismo: Estructura y desarrollo, México, McGraw-Hill.

Secretaría de Desarrollo Social (2005), Vivienda: Evidencia del cambio, México, Fondo de Cultural Económica, collection «Editorial del Gobierno del Cambio ».

Secretaría de Turismo-Instituto Nacional de Estadística, Geografía e Informática (SECTUR-INEGI) (2003), Turismo interno: Los viajes de los residentes en México dentro del país, México, SECTUR.

Torres, Rebecca M., et Janet D. Momsen (2005), "Gringolandia: The construction of a New Tourist Space in Mexico ", Annals of the Association of American Geographers, vol. 95, n०2, p. 314-335.

Turner, Louis, et John Ash (1991), La horda dorada: El turismo internacional y la periferia del placer, Madrid, Endymion.

Youden, John (2006), "Real Estate Trends 20062007 », Prudential California Realty, Vallarta. Division, [http://www.prurealtypv.com/RET rends.html].

\section{Sites Internet consultés}

Pour les données sur le Mexique: [datatur. sectur.gob.mx].

Pour les données internationales: [www.unwto.org].

Toutes nos données proviennent d'une consultation faite le 27 décembre 2006. 